\title{
SIMBIOSIS: REHABILITACIÓN ENERGÉTICA UNIDAD VECINAL DE MATUTE
}

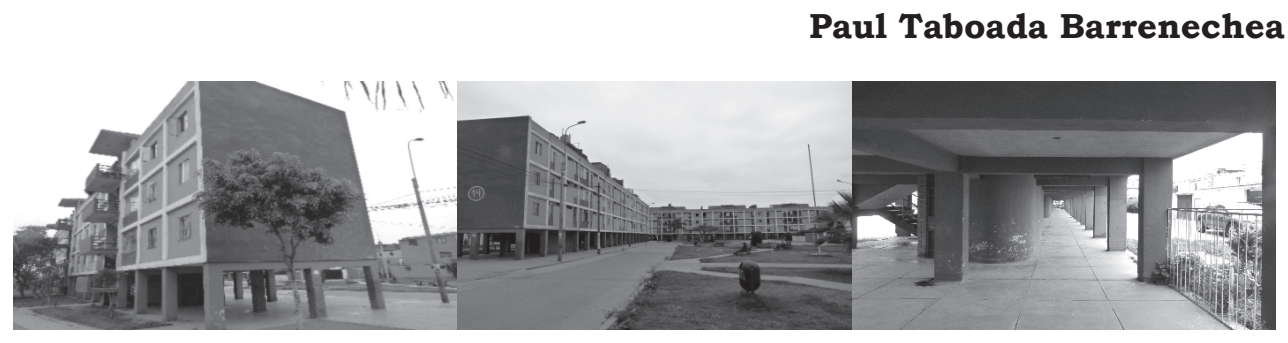

\section{Resumen}

El presente artículo se realizó con la finalidad de conocer y poner en práctica nuevas propuestas en base a tendencias arquitectónicas que incorporan la tecnología contemporánea para proyectar con el medio natural. Esta nueva tendencia considera a los elementos climáticos para proponer hábitats más sostenibles, ofreciendo mejor calidad de vida a los núcleos familiares que lo componen. A partir del concurso de Solar Declathon 2014, se tomó en cuenta el proyecto de Symbcity por tener características similares de vivienda en lo que refiere a bloques de edificios multifamiliares y realidad social. Según esta elección se tomaron en cuenta los aspectos tecnológicos utilizados en Symbcity, para ser considerados en un proyecto similar en nuestro medio, proponiendo nuevos aportes tecnológicos que vayan de acorde a nuestra realidad.

Palabras clave: Tecnología; Hábitat; Simbiosis.

\section{Abstract}

The following article was performed in order to learn and implement new proposals based on architectural trends that incorporate contemporary technology to project to the environment. This new trend to the elements considered to propose more sustainable habitat, offering better quality of life for families who 
compose it. From Solar Declathon Contest 2014 draft Symbcity was taken into account for housing have similar characteristics when it comes to blocks of multifamily buildings and social reality. According to this choice took into account technological Symbcity used to be considered in a similar project in our country, proposing new technological contributions that suit according to our reality.

Keywords: Tecnology; Habitat; Symbiosis.

\section{INTRODUCCIÓN}

En América latina la consideración de una política energética es aún escasa, siendo así que el suministro energético forma parte de la seguridad esencial de una nación.

Durante las últimas décadas, el sector vivienda en el Perú se ha venido desarrollando de manera desordenada e improvisada, implementando mecanismos deficientes e inadecuados para la concepción de las edificaciones en el ámbito de la eficiencia energética e hídrica. Esto lo convierte en un país insostenible, ya que muchos de los insumos utilizados para la construcción y post ocupancia se basa en un modelo de extracción y agotamiento de recursos que lo derivaría a la escasez de los mismos y afectaciones a los distintos ecosistemas. La necesidad de la población para acceder a una vivienda cercana a las zonas de mayor actividad económica, ha generado en su mayoría la toma de terrenos públicos o privados por parte de la población sin un control exhaustivo por parte de las autoridades. En nuestro medio, el sector habitacional es mayormente Informal, siendo ajeno a las formalidades administrativas, por lo que tiene como resultante la producción de viviendas improvisadas. La vivienda en el Perú y mayoritariamente en la capital presenta malas condiciones de habitabilidad, de precariedad habitacional y de localización de la vivienda en lugares inadecuados, debido tanto a la situación de pobreza en la que vive gran parte de la población, así como a las inadecuadas politicas públicas que no han tomado en cuenta esta situación. Por esto, se podria afirmar que actualmente los programas de vivienda no se interesan por el mantenimiento ni mejoramiento del parque habitacional sino por edificar viviendas nuevas con intermediación financiera de la banca comercial.

\section{CAMPO DE ESTUDIO}

La vivienda debe ofrecer un ambiente adecuado y propicio para el desenvolvimiento diario del núcleo familiar, ya que involucra el bienestar, el aspecto físico, psicológico y social del hombre. Por esta razón, debe ser proyectada de manera objetiva para lograr que la misma sea eficiente en el ámbito de la sostenibilidad, con una constante comunicación con el medio ambiente para garantizar la calidad de vida del usuario. El excesivo uso de los recursos energético e hídrico se convierte en un grave problema que se manifiesta en la degradación medioambiental, las limitaciones en la economía de las familias, en las zonas más pobres. 
La Unidad Vecinal $N^{\circ} 5$ de Matute, ubicada en el distrito de La Victoria, donde destacan los edificios de vivienda de 4 niveles y viviendas unifamiliares, se convirtió en ejemplo urbanístico de las tipologías de unidades vecinales de los años 50 durante el gobierno del general Odría. Hoy, a pesar de que la unidad vecinal continúa manteniendo su tipología original, se evidencia una transformación en las viviendas, hechas de manera improvisada, como lo demuestran las innumerables ampliaciones y alteraciones practicadas durante los últimos años. A ello se suma la incorporación de nuevas tecnologías en cada una de las viviendas que, a lo largo de los años, desde su creación, se ha manifestado en un aumento desproporcionado en cuanto al uso de los recursos energético e hídrico, por lo que se ha detectado que en los bloques de vivienda, el consumo mensual del recurso hídrico es aproximadamente de 50 nuevos soles con un volumen promedio de 9 metros cúbicos de agua potable. Del mismo modo, el consumo energético es de 120 nuevos soles mensuales, con un consumo promedio de 250 kW.h. Dichos resultados se obtuvieron en base a los consumos mensuales de las familias que habitan en el block número 14 de la unidad vecinal, conformado por 18 unidades de vivienda a razón de un promedio de 4 residentes por departamento.

\section{IMPORTANCIA $Y$ JUSTIFICA- CIÓN DEL ESTUDIO}

En el Perú son escasas las edificaciones que consideran tecnologías sostenibles que puedan aportar soluciones al problema medioambiental que se ha venido dando a lo largo de las últimas décadas.

La Unidad Vecinal Número 5 de Matute, considerando la importancia de ser uno de los primeros conjuntos residenciales proyectados en Latinoamérica, fue diseñado en el año 1952 por el arquitecto Santiago Agurto. Entre los años de 1953 y 1954 se construyó la primera etapa según el plan original, encargándose una década más tarde al arquitecto Enrique Ciriani terminar el diseño del conjunto. La intervención de ambos arquitectos tiene una clara diferencia, pero se puede apreciar que los edificios de la unidad vecinal dialogan entre sí y se reconocen como parte de una totalidad. La Unidad Vecinal número 5 de Matute en conjunto, se lee como una unidad y se puede distinguir de manera clara en la trama urbana de Lima.

Debido al crecimiento de la población, la necesidad de contar con mayores espacios y la aplicación de las nuevas tecnologías, resultado de la globalización ocurrida durante los últimos años, se ha producido un deterioro medioambiental y calidad de vida del residente en nuestro medio. En la Unidad Vecinal $N^{\circ} 5$ de Matute, la intervención de las unidades de vivienda del bloque número 14, por intermedio de la aplicación de la eficiencia energética e hidrica, trae consigo beneficios como mejoras en la salud (especialmente en niños y ancianos por la ausencia de frío en los ambientes), mejora en la percepción de la tempera- 
tura, mejor ventilación e iluminación, mayor tiempo de permanencia en la vivienda (mayor actividad en familia), mayor comodidad, menor consumo y derroche de energía y agua, y mayor presupuesto disponible en las familias para cubrir sus necesidades básicas. Así mismo, se considera que el menor consumo energético evita la degradación de muchos ecosistemas y aumenta la disponibilidad de energía para otros sectores.

Los resultados de la presente investigación servirán para plantear soluciones mediante la rehabilitación energética e hídrica del bloque número 14 de la Unidad Vecinal $\mathrm{N}^{\circ} 5$ de $\mathrm{Ma}$ tute, y así mejorar la calidad de vida de los residentes, aportando con el cuidado medioambiental, considerando tecnologías limpias y sostenibles. Entendiendo que mediante un correcto diagnóstico espacial del conjunto, el conocimiento del grado del problema y de la aplicación de las tecnologías actuales sostenibles, se podrá proponer la tecnología adecuada que mitigue el impacto ambiental generado por las unidades de vivienda.

\section{SIMBIOSIS: U.V. MATUTE}

Como respuesta a la problemática de la vivienda en Lima metropolitana y los nuevos barrios emergentes en cuanto al uso de la energía, se propone la adaptabilidad de nuevos módu- los de vivienda eficientemente energéticas (Huésped) en la azotea de un bloque de viviendas convencionales (Anfitrión), con lo cual se logrará la rehabilitación total del conjunto con energías limpias en una solución denominada simbiosis.

La estrategia consiste en adquirir la última planta del edificio anfitrión mediante una compra-venta a la junta de propietarios. Con el dinero recaudado por la venta de los huéspedes, este servirá para rehabilitar todo el conjunto. El huésped logrará proporcionar energía limpia a todo el conjunto, logrando la eficiencia energética del mismo y un ahorro en la economía de las familias.

\section{DESCRIPCIÓN DEL ANFITRIÓN}

El anfitrión elegido, corresponde a uno de los bloques de departamentos de $90 \mathrm{~m} 2$, donde se consideran 18 departamentos distribuidos en 3 pisos (6 departamentos por piso). Se ha obtenido un cálculo de 4 personas por núcleo familiar, por lo que el total de ocupantes del anfitrión es de 72 personas. La altura aproximada del bloque es de 11 metros, considerando que cada planta tiene una altura de 2.50mts. y 0.20mts de losa. Así mismo, se ha detectado que actualmente en el anfitrión se hace mal uso del agua y energía debido al consumo excesivo de las familias. 
Fig. 1. Unidad Vecinal $N^{\circ} 5$ de Matute. Ubicación del anfitrión a intervenir.

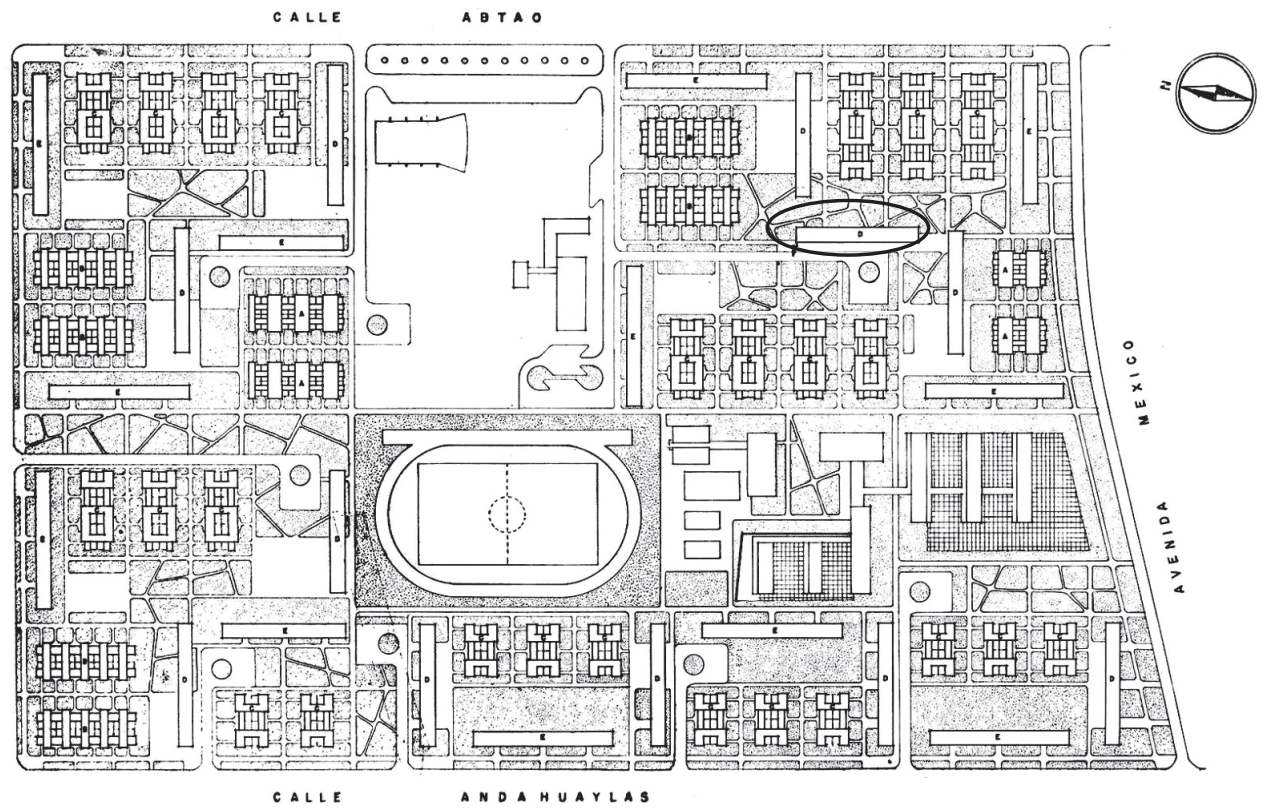

Fuente: Imagen obtenida del Análisis Unidad Vecinal N5 de Matute - Lima.

\section{DESCRIPCIÓN DEL HUÉSPED}

El huésped se encontrará ubicado en la última planta del bloque número 14 , considerándose su ubicación en dirección este-oeste con respecto a su fachada principal, donde la incidencia solar será preponderante. La superficie con la que cuenta la planta a intervenir es de aproximadamente $525 \mathrm{~m} 2(7.50 \times 70)$. El módulo huésped ocupará un área de $75 \mathrm{~m} 2(7.50 \times 10)$ que será repetido a lo largo de la superficie del bloque (aproximadamente 6 módulos de vivienda huésped). 
Fig. 2. Unidad Vecinal $N^{\circ} 5$ de Matute. Ubicación del módulo huésped en la última planta del anfitrión.

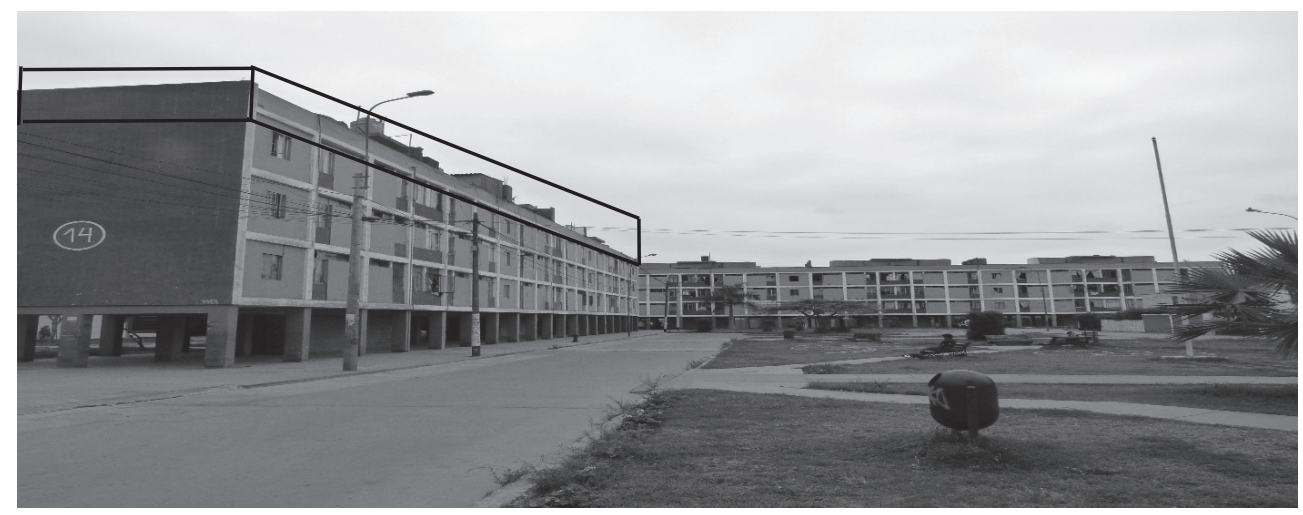

(Fuente: Imagen obtenida por el autor)

Fig. 3. Distribución del módulo huésped.

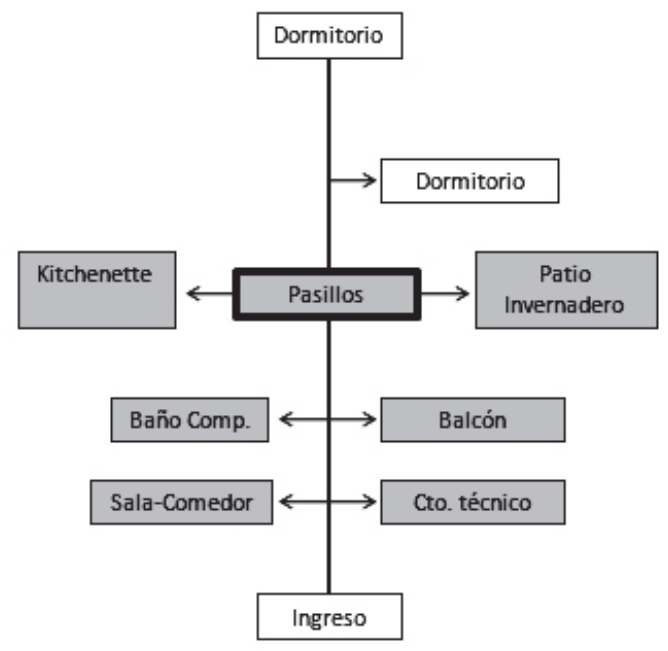

(Fuente: Esquema diseñado por el autor.)

\section{DESCRIPCIÓN DE LA SIMBIOSIS}

La simbiosis formulada se basa en que los módulos de viviendas hués- ped rehabilitarán energéticamente al anfitrión, como se indica en el esquema. 
Fig. 4. Esquema de simbiosis en la Unidad Vecinal $N^{\circ} 5$ de Matute.

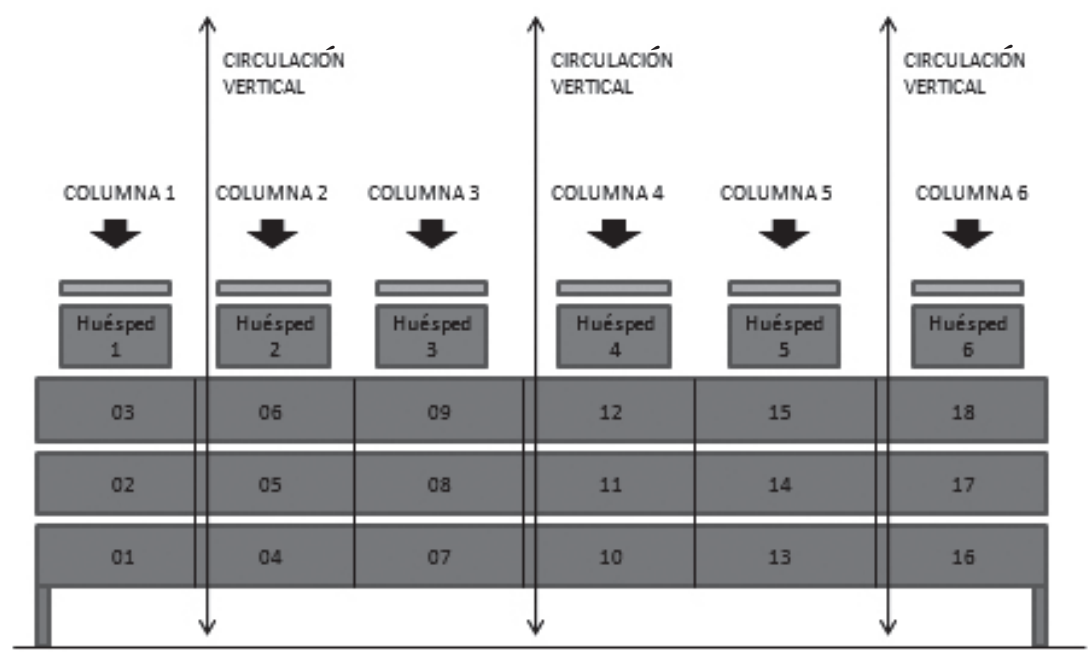

(Fuente: Esquema diseñado por el autor.)

\section{REPLANTEOS EN EL EDIFICIO ANFITRIÓN}

Los bloques cuentan en la última planta con un acceso de circulación vertical marcada por dos elementos en sus lados de uso común. Para lograr tener una superficie libre, se ha propuesto la eliminación de estos 2 espa- cios que acompañan al remate de las escaleras y dejar solo el acceso vertical con su respectivo alero. Para el acceso al módulo huésped, se está considerando la circulación vertical existente aprovechando su conexión con la última planta.

Fig. 5. Imagen de la circulación vertical del módulo anfitrión.

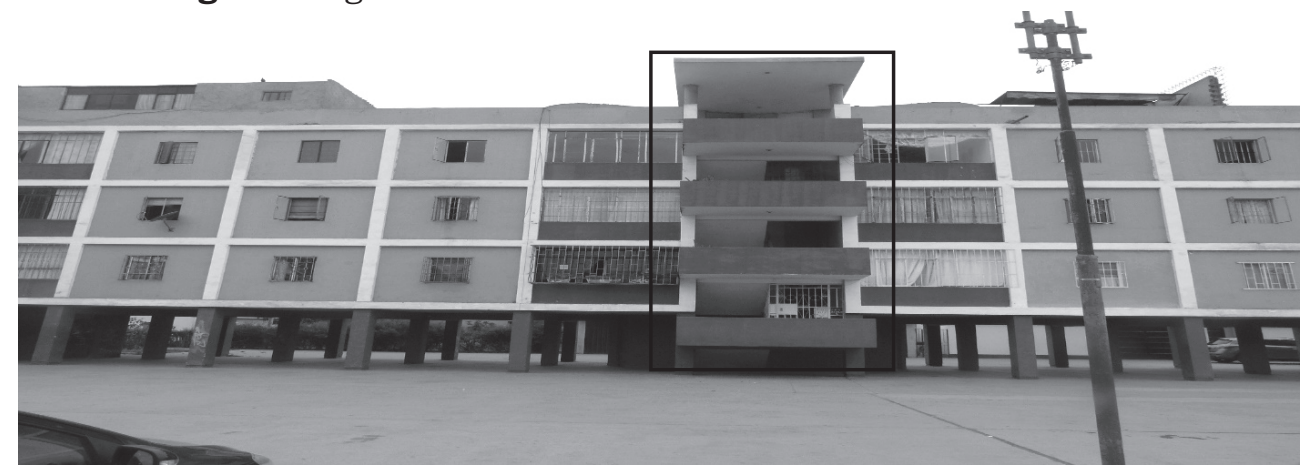

(Fuente: Imagen obtenida por el autor.) 


\section{ESTRUCTURA}

El reglamento indica que el piso de cada planta de un edificio debe soportar una carga de $200 \mathrm{~kg} / \mathrm{m}^{2}$, por lo cual se procedería a realizar un estudio de cargas mediante diamantinas, de no contar con los planos de estructuras del edificio para poder instalar al huésped. La base del huésped será de madera estructural de tipo C-Huayruro (se puede utilizar como madera utilitaria de construcción, donde se combine resistencia y facilidad de trabajo como corte, ensamblaje, montaje, etc.). Estará hecha en base de vigas estandarizadas con una separación de $0.80 \mathrm{~m}$ entre cada una de ellas, con un peralte de $0.30 \mathrm{~cm}$, por lo cual el módulo se adaptará de manera flexible a su anfitrión, empernándose directamente a él. El piso será de madera machihembrada e irá sobrepuesto y empernado a la trama de vigas de madera. El módulo irá empernado al anfitrión y elevado $30 \mathrm{~cm}$ del piso para evitar el contacto directo, creando una junta sísmica que servirá a la vez para mantener cálida la vivienda por el piso al ingreso de los vientos.

Fig. 6. Esquema de encuentro entre el anfitrión y el huésped.

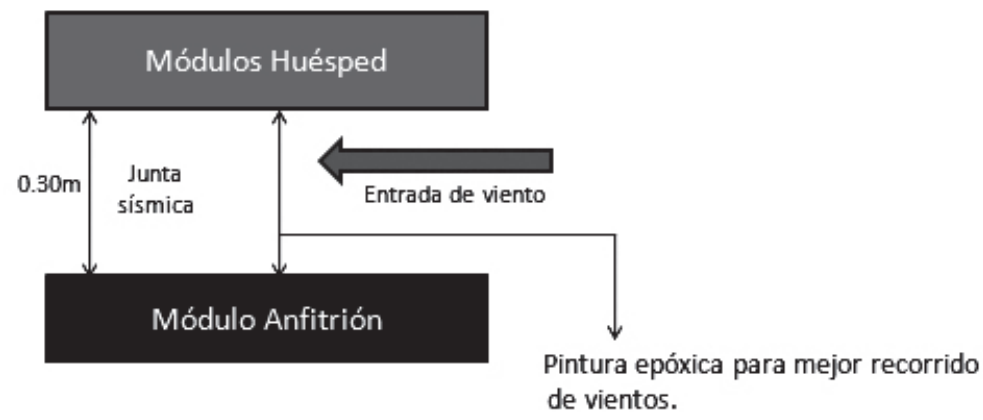

(Fuente: Esquema diseñado por el autor.)

\section{MUROS Y TABIQUERIAS}

Los tabiques interiores se realizaran con un producto para la construcción de cerramientos de la ONG Luz y Esperanza, que se trata de un sándwich de fibrocemento al exterior y cartón corrugado que tiene una transmitancia térmica, según test de la UNI, de $0.5 \mathrm{w} / \mathrm{m} 2 \mathrm{k}$. Para las tabiquerías exteriores se utilizaran paneles SIP, hechos a modo de sándwich con planchas de OSB al exterior y poliestireno al interior, lo que le otorga aislamiento térmico. Como recubrimiento exterior se puede considerar superboard o smartside. El OSB proviene de la madera reciclada y, además, el panel es estructuralmente rígido, lo que lo convierte en una opción sismo resistente, térmico y acústico. El panel SIP no necesita una estructura de apoyo, solo la conexión. El espesor dependerá de cuánto aislamiento térmico se desee. Para Lima, un mínimo de $5 \mathrm{~cm}$ de po- 
liestireno sería lo apropiado. El espesor total del muro es el espesor del aislamiento y las dos caras de OSB. Estos sistemas constructivos cuentan con aprobación de SENCICO como sistemas constructivos no convencionales.

Fig. 7. Paneles SIP.

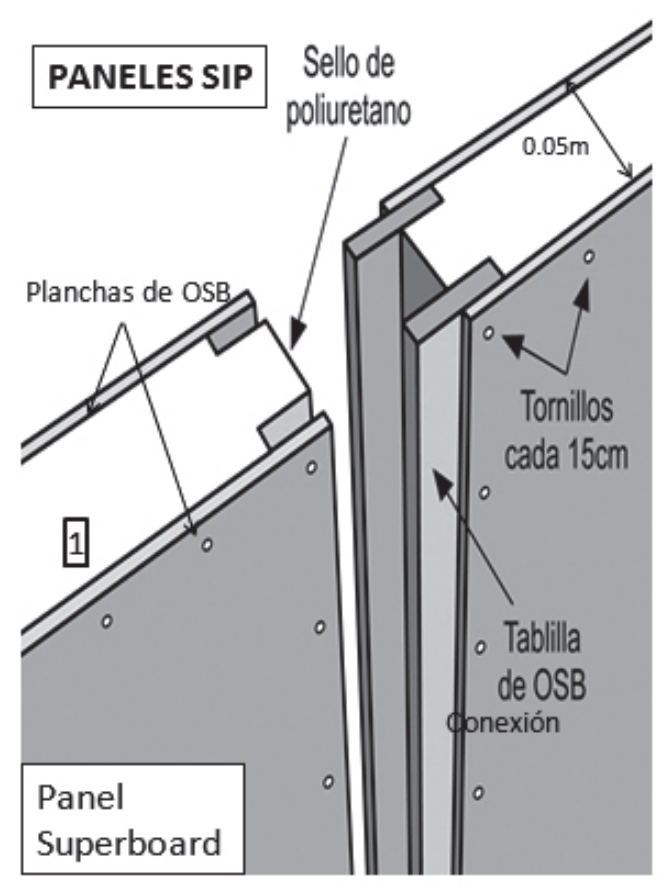

(Fuente: www.catalogoarquitectura.cl)

\section{PATIO INVERNADERO}

Al interior del patio invernadero se propondrá un área verde de intensidad media que mantendrá fresco el espacio en verano y dará confort térmico en invierno, al igual que la superficie vidriada, que serán manejadas dependiendo de la estación y el grado de confort térmico que se requiera al interior del módulo huésped. Para evitar costos elevados en el mantenimiento del área verde de intensidad media, se considerará un macetero rectangu- lar de $6 \mathrm{~m} \times 50 \mathrm{~cm}$, donde se incluirán una geomembrana en su base y 4 conexiones que servirán de desfogue del agua de regadío. Cada desfogue con un colador para evitar que el abono o la tierra se fuguen provocando atoros en las tuberias. El área verde de intensidad media se hará con la especie Pyracantha Coccinea, siendo una especie muy dúctil prestándose prácticamente a cualquier forma que se le requiera dar. 
Fig. 8. Ubicación del patio invernadero.

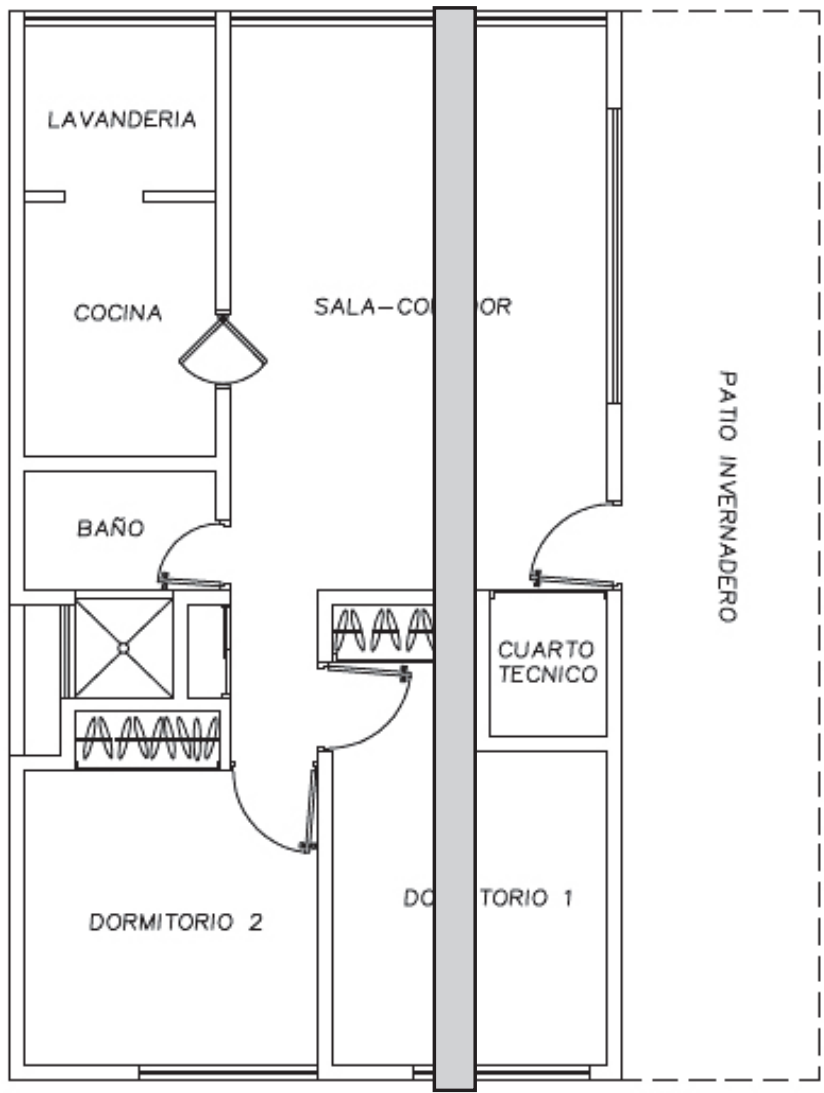

(Fuente: plano de distribución diseñado por el autor)

\section{Cálculo de paneles solares para}

\section{1 departamento huésped}

Para el cálculo de paneles solares se ha utilizado la Calculadora para Dimensionar Sistemas Fotovoltaicos de Delta Volt SAC Energías Renovables (deltavolt.pe/calculo-solar), empresa que promueve aplicaciones usando fuentes de energias renovables, ofreciendo soluciones de energía solar, eólica e hidráulica. Los aparatos considerados para el huésped son: 15 leds distribuidos en todo el módulo, 1 televisor, 1 radio, 1 computadora, 1 campana extractora, 1 refrigeradora, 1 microondas, 1 lavadora y artefactos provisionales.

La potencia requerida es de 1250 vatios (W) a más. Para cada huésped se requieren 2 paneles de 130 watts pico cada uno, que da un resultado de 1430 vatios. Debido a que la radiación en Lima es de 5.5 horas $/ \mathrm{rad}$. X 130 wpico que es la capacidad de 1 panel, entonces da una potencia de 715 vatios, por lo que se requerirían 2 paneles para obtener 1,430 vatios, que es más de lo que se requiere como indica 
el cálculo. Las dimensiones de cada panel son de $1.28 \times 0.669 \times 35 \mathrm{~mm}$ con un peso de $10.5 \mathrm{~kg}$ de la marca Dr. Solar España. El costo de cada panel es de 175 euros (S/. 643.90 aproximadamente) $+30 \%$ por aranceles, envío, instalación, etc.

\section{Cálculo de paneles solares para 1 departamento anfitrión}

Los aparatos considerados para la vivienda anfitrión son: 15 leds distribuidos en todo el modulo, 1 televisor, 1 radio, 1 computadora, 1 campana extractora, 1 refrigeradora, 1 microon- das, 1 lavadora y artefactos provisionales. Las áreas consideradas en el departamento anfitrión son 3 dormitorios, sala comedor, cocina, patio-lavandería, baño y pasillo.

La potencia requerida es de 1250 vatios (W) a más. Para cada departamento anfitrión se requieren 2 paneles de 130 watts pico cada uno, que da un resultado de 1430 vatios. Al igual que el caso del huésped, se requeririan 2 paneles para obtener 1,430 vatios, que es más de lo que se requiere como indica el cálculo.

\section{Cálculo de paneles solares para el conjunto}

Fig. 9. Resultados del cálculo de paneles solares para el conjunto.

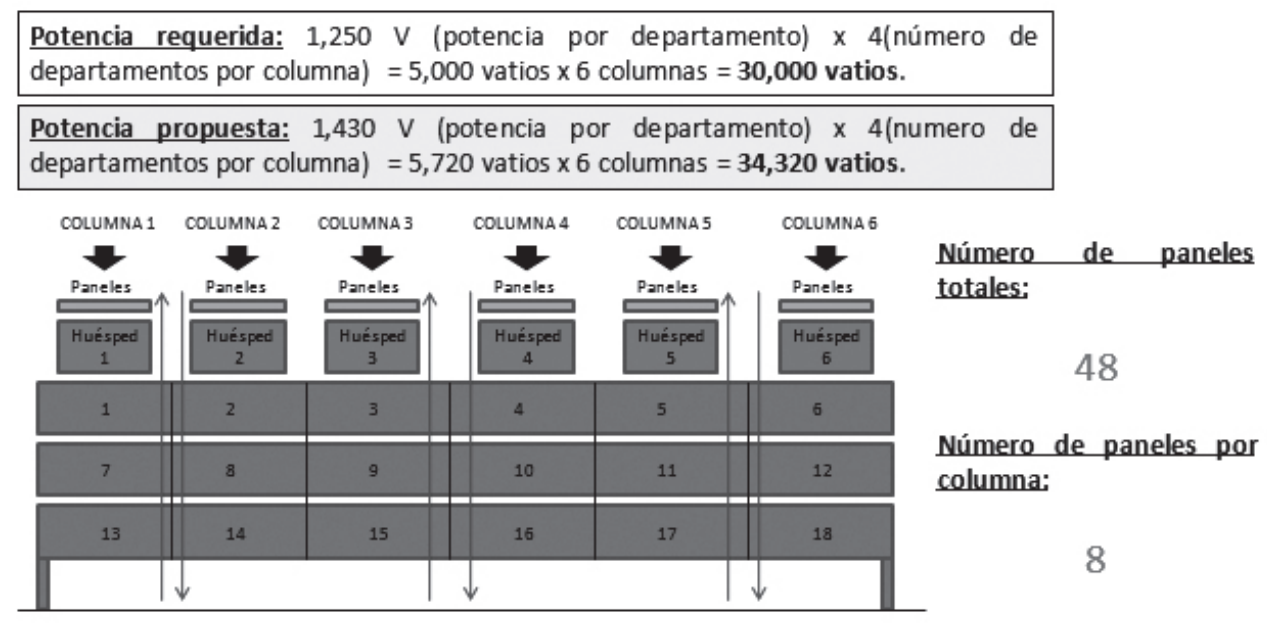

(Fuente: Esquema diseñado por el autor.) 


\section{Sistema de reciclaje de agua para el módulo huésped}

El sistema de reciclaje de aguas grises y negras para cada módulo huésped se dará de la siguiente manera.

Fig. 10. Sistema de reciclaje de agua para el modulo huésped.

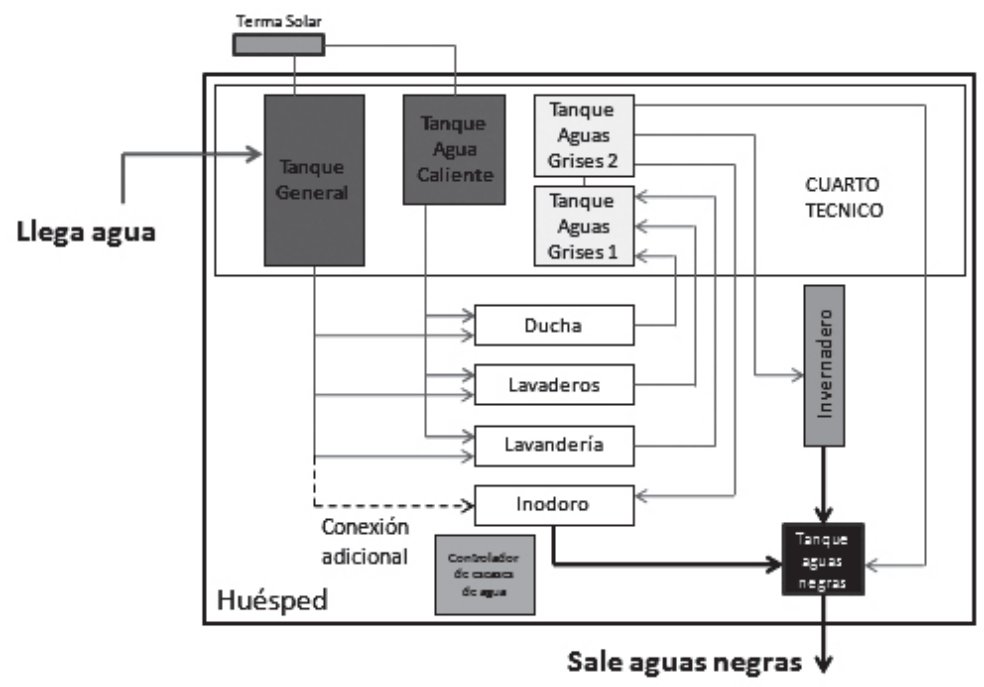

(Fuente: Esquema diseñado por el autor.)

\section{Sistema de reciclaje de agua para el módulo anfitrión}

El sistema de reciclaje de aguas grises y negras para cada módulo anfitrión estará dado de la siguiente manera:

Fig. 11. Sistema de reciclaje de agua para el módulo anfitrión.

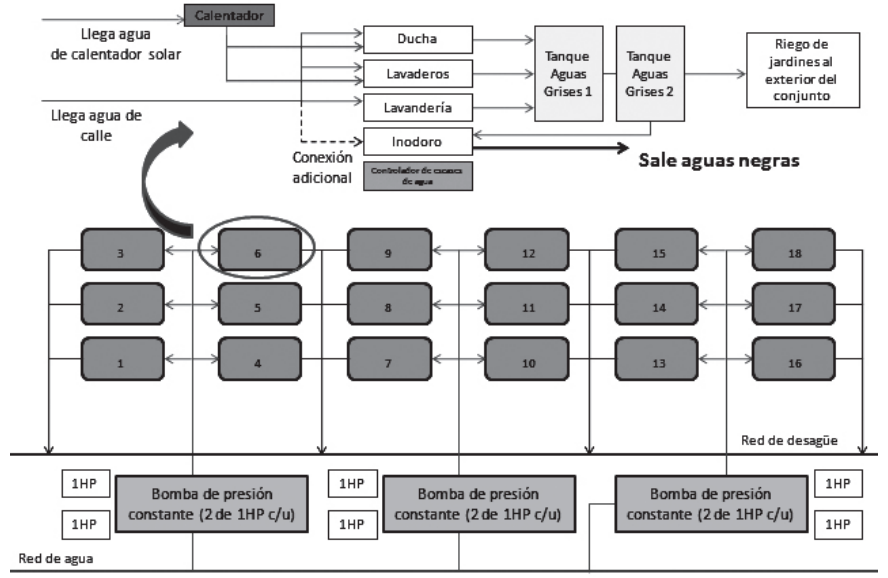

(Fuente: Esquema diseñado por el autor.) 


\section{Sistema de agua caliente por ca- lentadores solares}

Según el RNE, la dotación de agua diaria para una vivienda de $90 \mathrm{~m} 2$ es de 1,500 1ts. La dotación de agua caliente será el $20 \%$ de la dotación diaria, que serían 300 lts. de agua caliente al día.

En el ejemplo propuesto a continuación, la dotación de agua caliente se dará mediante el uso de 1 sistema de paneles solares, que cubrirá la demanda para 6 departamentos el bloque anfitrión, por lo que se necesitaran 2 sistemas de paneles solares más, para satisfacer la demanda de los otros 12 departamentos del conjunto. La marca del calentador solar a considerarse será Termoinox, con paneles Omega Plus de Delta Volt SAC, modelo TES 2000. El tamaño de los paneles es de $2.12 \times 0.90 \mathrm{~m}$, siendo en total 12 paneles para el primer sistema. La superficie ocupada por el sistema para la dosificación de los 6 departamentos mencionados como ejemplo, es de $22.90 \mathrm{~m}$, teniendo una capacidad de 2,000 litros, donde 300 litros es la ración de agua caliente por día que se le dará a cada departamento. Esto multiplicado por 6 departamentos que conforman el primer sistema, da como resultado la dotación de 1,800 litros de agua caliente al día, por lo que el sistema elegido cubrirá la demanda de los 6 departamentos del sistema 1. Se han considerado 3 sistemas de paneles, por lo que cada uno satisface a 6 departamentos del anfitrión. El costo por sistema es de S/. 24,700, incluido el IGV.

Fig. 12. Sistema de agua caliente por calentadores solares para los departamentos anfitrión.

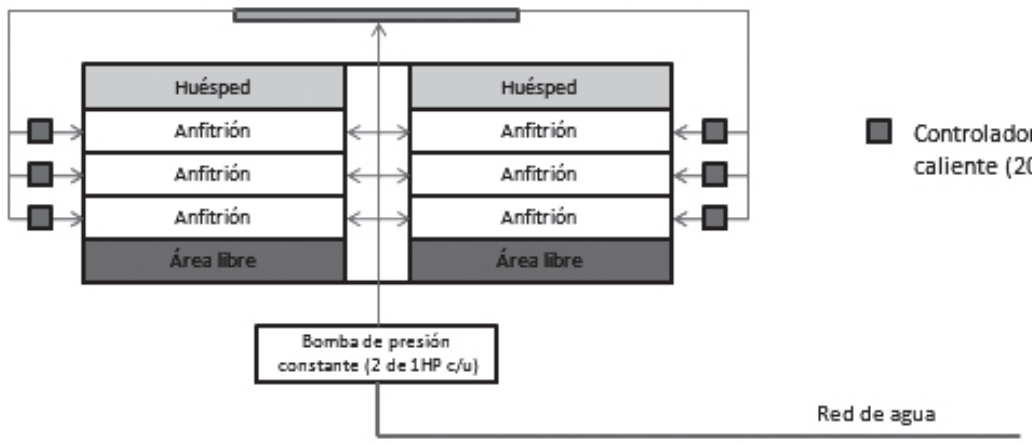

(Fuente: Esquema diseñado por el autor.) 
Fig. 13. Sistema de agua caliente por calentadores solares para los departamentos huésped.

Para la dotación de agua caliente para los departamentos huésped, se utilizó el mismo artificio.

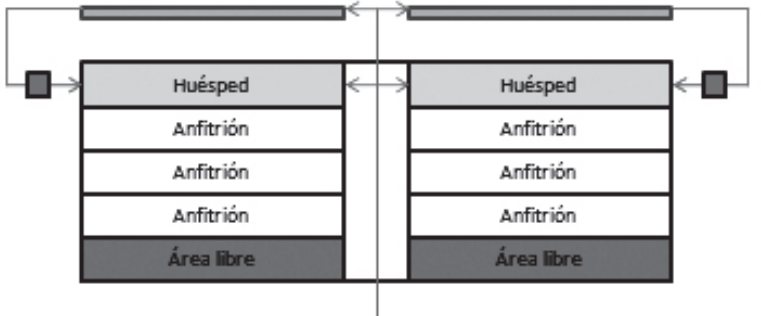

Leyenda

Bomba de presión

constante $\{2 \mathrm{de} 1 \mathrm{HP} c / \mathrm{u}\}$

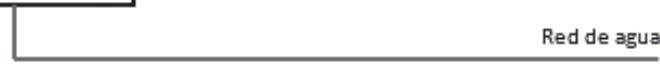

(Fuente: Esquema diseñado por el autor.)

\section{SISTEMA DE AUTOMATIZACIÓN}

Fig. 14. Sistema de automatización del conjunto.

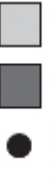

Espacios con sensor de presencia

Espacios con sensor de fuga de agua (a $1 \mathrm{~cm}$ del piso)

untos de luz ahorradores de 18 watts

1 ahorrador aprox. 800 lúmenes)

Controlador de dotación de agua caliente (20\%)

Dpto. Anfitrión 90m2

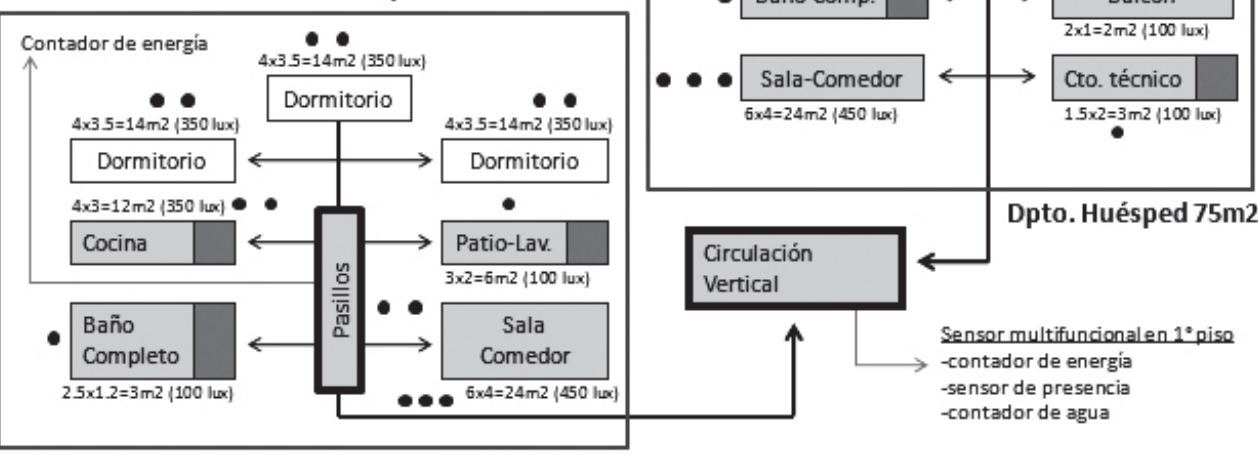

(Fuente: Esquema diseñado por el autor.) 


\section{CONCLUSIONES}

La suburbanización se ha convertido en un gran problema en cuestiones de energía, sobre todo en el área metropolitana de Lima. La concepción de un habitat más eficiente debe resolver este fenómeno, ya que la mayoria de las ciudades representa un desperdicio enorme de energía.

Como respuesta a la problemática de la vivienda en Lima Metropolitana y los nuevos barrios emergentes en cuanto al uso de la energía, se propuso la adaptabilidad de nuevos módulos de vivienda eficientemente energéticas denominados huésped, a un bloque de viviendas convencionales denominados anfitrión, por lo que se logrará la rehabilitación total del conjunto con energías limpias, denominado simbiosis.

\section{RECOMENDACIONES}

Se hace necesaria la evaluación del estado energético que ofrece una construcción convencional para poder conocer su impacto para con el medio ambiente.

Proponer en las edificaciones las estrategias correspondientes al adecuado uso de las tecnologias limpias con la finalidad de considerar la rehabilitación de las mismas.

Considerar las herramientas necesarias que complementen una adecuada rehabilitación energética de una edificación convencional, como lo es la aplicación de la automatización en las mismas.

La labor del arquitecto es hacer de conocimiento del usuario la importancia de la aplicación de todas estas tecnologías para lograr un habitat más amigable con el medio ambiente $y$, a la vez, la consideración de un ahorro en la economía del núcleo familiar. 


\section{REFERENCIAS BIBLIOGRÁFICAS}

Habitar (2012). Unidad Vecinal Matute, Análisis y Reconstrucción [online]. Accesible en <http://habitar-arq.blogspot.com/2012/06/unidad-vecinal-matute.html> [acceso 20 de febrero de 2015].

Lazarte H. (2014). Adaptación de las Viviendas Existentes a la Eficiencia Energética, Seminario de Eficiencia Energética, Perú, Universidad Ricardo Palma.

García J. (1982). El Reúso de Agua y sus Implicaciones [online]. Accesible en <www.mty.itesm.mx/die/ddre/transferencia/Transferencia52/eli4-52.html> [acceso 15 de marzo de 2015].

Habitar (2012). Unidad Vecinal Matute, Análisis y Reconstrucción [online]. Accesible en <http://habitar-arq.blogspot.com/2012/06/unidad-vecinal-matute.html> [acceso 20 de febrero de 2015].

Revista Invi (2005). El Problema de la Vivienda en el Peru, Retos y Perspectivas [online]. Accesibleen<http://www.revistas.uchile.cl/index.php/INVI/article/viewArticle/8818> [acceso 16 de febrero de 2015].

Ugarte F. (2014). Implementación de un Sistema de Paneles Fotovoltaicos (Solares) para Suministro de Energía Eléctrica en los Exteriores de un Condominio, Ecuador, Universidad Católica de Santiago de Guayaquil.

Eco Inteligencia (2013). Symbcity, La propuesta de Plateau Team para un Hábitat Humano Ecoeficiente [online]. Accesible en <http://plateauteam.com/ symbcity/> [acceso 22 de octubre de 2014].

Sousa G. (2011). Rehabilitación Térmica, Energética y Ambiental, Para la Sustentabilidad de la Vivienda en México. México, Universidad Nacional Autónoma de México.

Plateau Team (2014). Symbcity House [online]. Accesible en <http://www. ecointeligencia.com/2014/03/symbcity-house-solar-decathlon-europe-2014/> [acceso 15 de octubre de 2014].

Kahatt S. (2014). Lima: Cinco Siglos de Orden y Caos. Breve Recuento de Crecimiento y Transformación Socio-Espacial, Perú, Iberoamérica. 